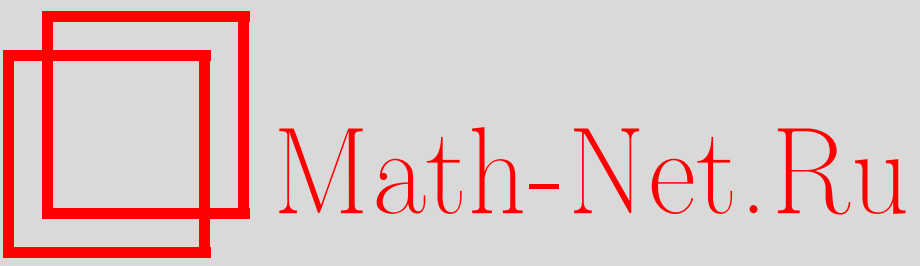

М. М. Гриненко, Новые структуры Мори на двойном пространстве индекса 2, УМH, 2004, том 59, выпуск 3, 163-164

DOI: https://doi.org/10.4213/rm745

Использование Общероссийского математического портала Math-Net.Ru подразумевает, что вы прочитали и согласны с пользовательским соглашением

http://www . mathnet.ru/rus/agreement

Параметры загрузки:

IP : 3.80 .253 .173

26 апреля 2023 г., 14:42:39 


\section{НОВЫЕ СТРУКТУРЫ МОРИ НА ДВОЙНОМ ПРОСТРАНСТВЕ ИНДЕКСА 2}

\section{М. М. ГРИНЕНКО}

Пусть $V$ - трехмерное неособое многообразие Фано основной серии индекса 2 и степени 2 над алгебраически замкнутым полем характеристики 0 . Такое многообразие часто назьвают двойным пространством индекса 2 , посколшку оно обладает морфизмом $\pi: V \rightarrow \mathbb{P}^{3}$ степени 2 , разветвленным над неособой квартикой $Q \subset \mathbb{P}^{3}$. Мы полагаем $\operatorname{Pic}(V)=\mathbb{Z} H, K_{V} \sim-2 H$ и $H^{3}=2$. Напомним, что прямой на многообразии Фано назьвается неособая рациональная кривая степени 1 (относителшно пересечения с порождающей группы Пикара). С каждой точкой $A \in V$, не лежащей над дивизором ветвления, связан единственный (всегда неприводимый) эффективньй дивизор $G_{A} \sim 2 H$ такой, что mult $_{A} G_{A}=3$; этот дивизор является квазисечением расслоения на эллиптические кривые, получаемого раздутием точки $A$ и сопряженной с ней относительно морфизма $\pi$ точки.

Будем говорить, что точка $A$, не лежащая над дивизором ветвления, является специальной, если для некоторой поверхности $S \in|H|$, содержащей точку $A$, кривая $\left.G_{A}\right|_{S}=l_{1} \cup l_{2} \cup l_{3} \cup l_{4}$ составлена из различных прямых; при этом $A$ будем назьвать 3- или 4-специальной в зависимости от того, чему равно число mult $\left._{A} G_{A}\right|_{S}$ : трем или четьрем. Отметим, что 3 -специальные точки расположены на некоторой алгебраической поверхности на $V$, а 4 -специальные точки - на некоторой кривой. В дальнейшем будем полагать, что поверхность $S$ неособа вдоль прямых $l_{i}$.

С каждой 3 -специальной точкой связана перестройка $V \rightarrow X$ на многообразие $\Phi$ ано $X=$ $X_{3,3} \subset \mathbb{P}\left(1^{5}, 2\right)$, являющееся полньм пересечением двух кубик, а с каждой 4-специальной точкой - перестройка $V \rightarrow U$ на расслоение $U / \mathbb{P}^{1}$ на поверхности дель Пеццо степени 2 с одним кратньм слоем, при этом $X$ и $U / \mathbb{P}^{1}$ лежат в категории Мори.

Перестройка $V \rightarrow X=X_{3,3} \subset \mathbb{P}\left(1^{5}, 2\right)$. Пусть точка $A \in S$ является 3-специальной, $\left.G_{A}\right|_{S}=l_{1} \cup l_{2} \cup l_{3} \cup l_{4}, A \notin l_{4}$. На поверхности $S$ (а это поверхность дель Пещо степени 2) выполняется $l_{i}$ ○ $l_{j}=1$ при $i \neq j$ и $l_{i}^{2}=-1$. Из конструкции инволюции Бертини, связанной с точкой $A$, легко следует существование единственной кривой $q$ степени 3 (относительно антиканонического дивизора) такой, что mult $_{A} q=2$. Далее, если раздуть точку $A$ на $V$, то собственньй прообраз $S$ высечет на исключительной плоскости некоторую прямую, которую мы обозначим $p$. Пара $(A, p)$ определяет взвешенное раздутие $\varphi_{1}: V_{1} \rightarrow V$ точки $A$ с весами $(1,1,2)$ и исключительным дивизором $E_{1}$, изоморфным квадратичному конусу; геометрически $\varphi_{1}$ может быть получено так: мы раздуваем точку $A$, затем прямую $p$ и стягиваем собственный прообраз исключительной плоскости. Заметим, что $V_{1}$ имеет циклическую фактор-особенность типа $\frac{1}{2}(1,1,1)$ в вершине конуса $E_{1}$.

Перестройка $V \rightarrow X$ является линком Саркисова типа II и получается последовательностью бирациональных преобразований

$$
V \stackrel{\varphi_{1}}{\stackrel{\psi_{1}}{\leftrightarrows}} V_{1} \stackrel{\psi_{1}}{\rightarrow} V_{2} \stackrel{\psi_{2}}{\rightarrow} V_{3} \stackrel{\varphi_{2}}{\longrightarrow} V_{4}=X
$$

где (всюду далее верхние индексы у кривых и дивизоров обозначают собственньй прообраз на соответствующем многообразии $\left.V_{i}\right)$ :

1) отображение $\psi_{1}$ является антифлипом $Ф$ ранчи (Francia), выполненным одновременно в кривых $l_{1}^{1}, l_{2}^{1}$ и $l_{3}^{1}$, нормальные пучки которых изоморфны $\mathscr{O}(-1) \oplus \mathscr{O}(-2)$ (отметим, что $S^{1}$ не проходит через фактор-особенность на $\left.V_{1}\right)$;

2 ) отображение $\psi_{2}$ является фолопом с центром в кривой $q^{2}$, имеющей нормалшньй пучок $\mathscr{O}(-1) \oplus \mathscr{O}(-1)$

Работа вьполнена при поддержке грантов РФФИ 02-01-00441, НШ-489.2003.1, Фонда содействия отечественной науке и государственной программы "Современные проблемы теоретической математики".

(C) М. М. ГРИненко 2004 
3) морфизм $\varphi_{2}$ является дивизориальньм стягиванием в категории Мори поверхности $S^{3}$ в особую точку типа $c D_{4}$.

Существование указанных отображений несложно проверяется при помощи так назьваемой “двухлучевой игры” на конусах Мори. Дополнительно укажем следующее. Поверхность $S^{3}$ является поверхностью дель Пещю степени 2 и получается из $S$ раздутием точки $A$ с последующим стягиванием собственных прообразов кривых $l_{1}^{1}, l_{2}^{1}, l_{3}^{1}$ и $q$, причем кривая $l_{4}^{3}$ обильна на $S^{3}$ и, более того, порождает экстремальньй луч конуса $\overline{N E}\left(V_{3}\right)$. По данньм $\left(V_{3}, S^{3}\right)$ стягивание $\varphi_{2}$ устанавливается однозначно ввиду результатов работы [1]. Можно показать, что $V_{4}=X$ является полным пересечением двух кубических гиперповерхностей в $\mathbb{P}\left(1^{5}, 2\right)$. Таким образом, $X$ является многообразием Фано индекса 1 с фактор-особенностью $\frac{1}{2}(1,1,1)$ и точкой типа $c D_{4}$.

Любопытно, но из существования перестройки $V \rightarrow X$ несложно вывести, что $V$ бирационально изоморфно трехмерной квартике с плоскостью и $c D_{4}$-точкой вне плоскости.

Перестройка $V \rightarrow U / \mathbb{P}^{1}$. Относительно поверхности $S$ и прямых $l_{i}$ на ней можно сказать то же, что и выше, с той лишь разницей, что теперь $A \in l_{4}$. Как и в предыдущем случае, выделим "бесконечно близкую" прямую $p$ (но только теперь еще и собственный прообраз $G_{A}$ содержит $p$ ) и соответствующее взвешенное раздутие $\varphi_{1}: V_{1} \rightarrow V$. Перестройка $V \rightarrow U$ является линком Саркисова типа I и разлагается в композицию

$$
V \stackrel{\varphi_{1}}{\longleftarrow} V_{1} \stackrel{\psi}{\rightarrow} V_{2}=U
$$

где $\psi$ является антифолипом $\Phi$ ранчи одновременно во всех кривых $l_{i}^{1}$. Существование такого антифлипа устанавливается так же, как и выше. Важное наблюдение заключается в том, что $G_{A}^{1} \sim$ $2 S^{1}$ и $G_{A}^{2} \cap S^{2}=\varnothing$. Отсюда немедленно вытекает существование морфизма $\rho: V_{2}=U \rightarrow \mathbb{P}^{1}$ такого, что $G_{A}^{2} \sim 2 S^{2} \sim F$, где $F$ - класс слоя $\rho$. Легко подсчитать, что $G_{A}^{2}$ будет поверхностью дель Пещю степени 2 , а $S^{2}$ - поверхностью дель Пецю степени 1 . Таким образом, $U / \mathbb{P}^{1}$ является расслоением на поверхности делш Пецо степени 2 с одним слоем кратности 2 с носителем $S^{2}$. Многообразие $U$ имеет пять фактор-особенностей типа $\frac{1}{2}(1,1,1)$, четыре из которых лежат в кратном слое, а одна - на $G_{A}^{2}$.

ЗАмечАниЕ 1 . Отображения $V \rightarrow X$ и $V \rightarrow U / \mathbb{P}^{1}$ позволяют легко построить примеры линейных систем $V$ с бесконечно близкими максимальньми особенностями, определяемшіми парами $(A, p)$ (точка и бесконечно близкая прямая). Для этого достаточно взять собственные прообразы обильных линейных систем с $X$ или $U$. Например, собственньй прообраз $\left|\mathscr{O}_{X}(2)\right|$ на $V$ будет подсистемой в $|6 H|$, имеющей кратность 5 в точке $A$ и кратность 5 вдоль $p$. В случае 4 -специальной точки собственный прообраз $\left|-K_{U}+F\right|$ даст подсистему в $|4 H|$ с кратностью 4 в точке $A$ и кратностью 3 вдоль $p$. Вероятно, если не считать $\mathbb{P}^{3}$, это первьй случай, когда удалось явным образом предъявить линейные системы с бесконечно близкими максимальньми особенностями на трехмерном проективном многообразии. Наконец, заметим, что вполне естественный список структур Мори на двойном пространстве индекса 2, предложенньй автором в работе [2], необходимо расширить с учетом новых структур, описанных в настоящей заметке.

\section{СПИСОК ЛИТЕРАТУРЫ}

[1] И. Ю. Фёдоров. Чисто логтерминальные раздутия индекса 2 // Матем. сб. 2004 (в печати). [2] M. M. Grinenko. Birational models of del Pezzo fibrations // math.AG/0209394 (to appear in London Math. Soc. Lecture Note Ser.).

Математический институт им. В. А. Стеклова РАН

E-mail: grin@mi.ras.ru
Принято редколлегией 01.04 .2004 